Commun. Fac. Sci. Univ. Ank. Ser. A1 Math. Stat.

Volume 68, Number 2, Pages 1895-1908(2019)

DOI: $10.31801 /$ cfsuasmas.472024

ISSN 1303-5991 E-ISSN 2618-6470

http://communications.science.ankara.edu.tr/index.php?series=A1

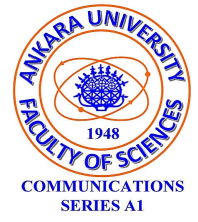

\title{
AUTOMATIC STRUCTURE FOR GENERALIZED BRUCK-REILLY *-EXTENSION OF A MONOID
}

\author{
EYLEM GÜZEL KARPUZ
}

\begin{abstract}
In the present paper, we study the automaticity of generalized Bruck-Reilly $*$-extension of a monoid. Under some certain situations, we prove that the automaticity of the monoid implies the automaticity of the generalized Bruck-Reilly *-extension of this monoid.
\end{abstract}

\section{Introduction and Preliminaries}

One of the most popular areas of computational algebra has recently been the theory of automatic groups. The description of a group by an automatic structure allows one efficiently to perform various computations involving the group, which may be hard or impossible given only a presentation. Groups which admit automatic structure also share a number of interesting structural and geometric properties [8]. Recently, many authors have followed a suggestion of Hudson [12] by considering a natural generalization to the broader class of monoids or, even more generally, of semigroups, and a coherent theory has begun to develop from the point of geometric aspects 21], computational and decidebility aspects [17, 18, 19, other notions of automaticity for semigroups [9, 10.

Many results about automatic semigroups concern automaticity of semigroup constructions. For instance, in 5] free product of semigroups, in 4 direct product of semigroups, in [7] Rees matrix semigroups, in [1, 3] Bruck-Reilly extension of monoids and wreath product of semigroups were studied. In [6], the author showed that a Bruck-Reilly extension $B R(S, \theta)$ of an automatic monoid $S$ is itself automatic

- if $S$ is finite (Theorem 5.1),

- if the mapping $\theta: S \rightarrow S$ sends every element of $S$ to $1_{S}$ (Theorem 5.2),

- if $\theta: S \rightarrow S$ is the identity mapping (Theorem 5.3),

- if $S$ is a finite geometric type automatic monoid and $S \theta$ is finite (Theorem $5.4)$.

Received by the editors: October 18, 2018; Accepted: February 28, 2019.

2010 Mathematics Subject Classification. Primary 20M05; Secondary 20M35, 68Q45.

Key words and phrases. Automatic semigroup, generalized Bruck-Reilly *-extension, presentation.

(C)2019 Ankara University Communications Faculty of Sciences University of Ankara-Series A1 Mathematics and Statistics 
These results and their proofs are reproduced in a survey article by Andrade et al. 11. In the present paper, by considering the results given in [6], we study on generalized Bruck-Reilly $*$-extension of a monoid of which presentation was firstly defined in [14. A generalized Bruck-Reilly *-extension was first introduced in [2]. Since then many research papers have been published see for example [13, 15, 16, 20. We prove the following results:

Theorem 4 If $T$ is a finite monoid then generalized Bruck-Reilly $*$-extension of $T$ is automatic.

Theorem 6 If $T$ is an automatic monoid and $\gamma, \beta: T \rightarrow H_{1}^{*} ; t \mapsto 1_{T}$ then generalized Bruck-Reilly *-extension of $T$ is automatic.

Theorem 7 If $T$ is an automatic monoid and $\gamma, \beta$ are identity homomorphisms of $T$ then generalized Bruck-Reilly $*$-extension of $T$ is automatic.

Theorem 10 Let $T$ be a finite geometric type automatic monoid and let $\gamma, \beta$ : $T \rightarrow H_{1}^{*}$ be homomorphisms. If T $\gamma, T \beta$ are finite then generalized Bruck-Reilly *-extension of $T$ is automatic.

Let $A$ be an alphabet. We denote by $A^{+}$the free semigroup generated by $A$ consisting of finite sequences of elements of $A$, which we call words, under the concatenation; and by $A^{*}$ the free monoid generated by $A$ consisting of $A^{+}$with the empty word $\epsilon$, the identity in $A^{*}$. For a word $w \in A^{*}$, we denote the length of $w$ by $|w|$. Let $S$ be a semigroup and $\phi: A \rightarrow S$ a mapping. We say that $A$ is a finite generating set for $S$ with respect to $\phi$ if the unique extension of $\phi$ to a semigroup homomorphism $\psi: A^{+} \rightarrow S$ is surjective. For $u, v \in A^{+}$we write $u \equiv v$ to mean that $u$ and $v$ are equal as words and $u=v$ to mean that $u$ and $v$ represent the same element in the semigroup. In other words $u \psi=v \psi$. We say that a subset $L$ of $A^{*}$, usually called a language, is regular if there is a finite state automaton accepting $L(\underline{5})$. To be able to deal with automata that accept pairs of words and to define automatic semigroups we need to define the set $A(2, \$)=((A \cup\{\$\}) \times(A \cup\{\$\}))-\{(\$, \$)\}$ where $\$$ is a symbol not in $A$ (called the padding symbol) and the function $\delta_{A}: A^{*} \times A^{*} \rightarrow A(2, \$)^{*}$ defined by

$\left(a_{1} \cdots a_{m}, b_{1} \cdots b_{n}\right) \delta_{A}= \begin{cases}\epsilon & \text { if } 0=m=n \\ \left(a_{1}, b_{1}\right) \cdots\left(a_{m}, b_{m}\right) & \text { if } 0<m=n \\ \left(a_{1}, b_{1}\right) \cdots\left(a_{m}, b_{m}\right)\left(\$, b_{m+1}\right) \cdots\left(\$, b_{n}\right) & \text { if } 0 \leq m<n \\ \left(a_{1}, b_{1}\right) \cdots\left(a_{n}, b_{n}\right)\left(a_{n+1}, \$\right) \cdots\left(a_{m}, \$\right) & \text { if } m>n \geq 0\end{cases}$

Let $S$ be a semigroup and $A$ a finite generating set for $S$ with respect to $\psi$ : $A^{+} \rightarrow S$. The pair $(A, L)$ is an automatic structure for $S$ (with respect to $\psi$ ) if

- $L$ is a regular subset of $A^{+}$and $L \psi=S$,

- $L_{=}=\{(\alpha, \beta): \alpha, \beta \in L, \alpha=\beta\} \delta_{A}$ is a regular in $A(2, \$)^{+}$, and

- $L_{a}=\{(\alpha, \beta): \alpha, \beta \in L, \alpha a=\beta\} \delta_{A}$ is a regular in $A(2, \$)^{+}$for each $a \in A$.

We say that a semigroup is automatic if it has an automatic structure. 
We say that the pair $(A, L)$ is an automatic structure with uniqueness (with respect to $\psi$ ) for a semigroup $S$, if it is an automatic structure and each element in $S$ is represented by an unique word in $L$ (the restriction of $\psi$ to $L$ is a bijection).

\section{Generalized Bruck-Reilly *-Extension}

Let $T$ be a monoid with $H_{1}^{*}$ and $H_{1}$ as the $\mathcal{H}^{*}$ - and $\mathcal{H}$ - class which contains the identity $1_{T}$ of $T$, respectively. Assume that $\beta$ and $\gamma$ are morphisms from $T$ into $H_{1}^{*}$. Let $u$ be an element in $H_{1}$ and let $\lambda_{u}$ be the inner automorphism of $H_{1}^{*}$ defined by $x \mapsto u x u^{-1}$ such that $\gamma \lambda_{u}=\beta \gamma$. Now we can consider $\mathbb{N}^{0} \times \mathbb{N}^{0} \times T \times \mathbb{N}^{0} \times \mathbb{N}^{0}$ into a semigroup by defining multiplication

$$
\begin{aligned}
& (m, n, v, p, q)\left(m^{\prime}, n^{\prime}, v^{\prime}, p^{\prime}, q^{\prime}\right)= \\
& \begin{cases}\left.\left(m, n-p+t,\left(v \beta^{t-p}\right)\left(v^{\prime t-n^{\prime}}\right), p^{\prime}-n^{\prime}+t\right), q^{\prime}\right) & \text { if } q=m^{\prime} \\
\left(m, n, v\left(\left(\left(u^{-n^{\prime}}\left(v^{\prime p^{\prime}}\right) \gamma^{q-m^{\prime}-1}\right) \beta^{p}\right), p, q^{\prime}-m^{\prime}+q\right)\right. & \text { if } q>m^{\prime} \\
\left.\left.\left.\left(m-q+m^{\prime}, n^{\prime-n}(v \gamma) u^{p}\right) \gamma^{m^{\prime}-q-1}\right) \beta^{n^{\prime}}\right) v^{\prime}, p^{\prime}, q^{\prime}\right) & \text { if } q<m^{\prime},\end{cases}
\end{aligned}
$$

where $t=\max \left(p, n^{\prime}\right)$ and $\beta^{0}, \gamma^{0}$ are interpreted as the identity map of $T$ and $u^{0}$ is interpreted as the identity $1_{T}$ of $T$. The monoid $\mathbb{N}^{0} \times \mathbb{N}^{0} \times T \times \mathbb{N}^{0} \times \mathbb{N}^{0}$ constructed above is called generalized Bruck-Reilly $*$-extension of $T$ determined by the morphisms $\beta, \gamma$ and the element $u$. This monoid is denoted by $G B R^{*}(T ; \beta, \gamma ; u)$ and the identity of it is the element $\left(0,0,1_{T}, 0,0\right)([20)$. For some information concerning semigroup theory such as $\mathcal{H}^{*}$ - and $\mathcal{H}$-Green relations, see [11].

In [14, the authors have obtained the following results.

Lemma 1. Suppose that $X$ is a generating set for the monoid $T$. Then

$$
\begin{aligned}
& \{(0,0, x, 0,0): x \in X\} \cup\left\{\left(1,0,1_{T}, 0,0\right) \cup\left(0,1,1_{T}, 0,0\right) \cup\left(0,0,1_{T}, 1,0\right)\right. \\
& \left.\cup\left(0,0,1_{T}, 0,1\right)\right\}
\end{aligned}
$$

is a generating set for the monoid $G B R^{*}(T ; \beta, \gamma ; u)$.

Theorem 2. Let $T$ be a monoid defined by the presentation $\langle X ; R\rangle$, and let $\beta, \gamma$ be morphisms from $T$ into $H_{1}^{*}$. Therefore the monoid $G B R^{*}(T ; \beta, \gamma ; u)$ is defined by the presentation

$$
\begin{aligned}
<X, y, z, a, b \quad ; \quad & R, \quad y z=1, \quad b a=1, \\
& y x=(x \gamma) y, \quad x z=z(x \gamma), \quad b x=(x \beta) b, \quad x a=a(x \beta)(x \in X), \\
& y b=u y, \quad y a=u^{-1} y, \quad b z=z u, \quad a z=z u^{-1}>.
\end{aligned}
$$

As a consequence of Theorem 2, we have the following result.

Corollary 3. Let $v$ be an arbitrary word in $X^{*}$. The relations

$$
\begin{array}{r}
y^{m} v=\left(v \gamma^{m}\right) y^{m}, \quad v z^{m}=z^{m}\left(v \gamma^{m}\right), \\
b^{n} v=\left(v \beta^{n}\right) b^{n}, \quad v a^{n}=a^{n}\left(v \beta^{n}\right), \\
y^{m} b^{n}=\left(u \gamma^{m-1}\right)^{n} y^{m}, \quad y^{m} a^{n}=\left(u^{-1} \gamma^{m-1}\right)^{n} y^{m},
\end{array}
$$




$$
b^{n} z^{m}=z^{m}\left(u \gamma^{m-1}\right)^{n}, \quad a^{n} z^{m}=z^{m}\left(u^{-1} \gamma^{m-1}\right)^{n}
$$

hold in $G_{B R}^{*}(T ; \beta, \gamma ; u)$ for all $m, n \in \mathbb{N}^{0}$. As a consequence, every word $w \in$ $(X \cup\{y, z, a, b\})^{*}$ is equal in $G B R^{*}(T ; \beta, \gamma ; u)$ to a word of the form $z^{m} a^{n} v b^{p} y^{q}$ for some $v \in X^{*}$ and $m, n, p, q \in \mathbb{N}^{0}$.

\section{Main Results}

We give the first result of this paper.

Theorem 4. If $T$ is a finite monoid then any generalized Bruck-Reilly-extension of $T$ is automatic.

Proof. Let $T=\left\{t_{1}, t_{2}, \cdots, t_{l}\right\}$ and let $\bar{T}=\left\{\overline{t_{1}}, \overline{t_{2}}, \cdots, \overline{t_{l}}\right\}$ be an alphabet in bijection with $T$. We define the alphabet $A=\{y, z, a, b\} \cup \bar{T}$ and the regular language

$$
L=\left\{z^{m} a^{n} \bar{t} b^{p} y^{q}: m, n, p, q \geq 0, \bar{t} \in \bar{T}\right\}
$$

on $A$. Defining the homomorphism

$$
\begin{aligned}
\psi: A^{+} & \rightarrow G B R^{*}(T ; \beta, \gamma ; u) ; \\
\bar{t} & \mapsto(0,0, t, 0,0), \\
y & \mapsto\left(0,0,1_{T}, 0,1\right), \\
z & \mapsto\left(1,0,1_{T}, 0,0\right), \\
a & \mapsto\left(0,1,1_{T}, 0,0\right), \\
b & \mapsto\left(0,0,1_{T}, 1,0\right),
\end{aligned}
$$

it is clear that $A$ is a generating set for $G B R^{*}(T ; \beta, \gamma ; u)$ with respect to $\psi$ and, in fact, given an element $(m, n, t, p, q) \in \mathbb{N}^{0} \times \mathbb{N}^{0} \times T \times \mathbb{N}^{0} \times \mathbb{N}^{0}$ the unique word in $L$ representing it is $z^{m} a^{n} \bar{t} b^{p} y^{q}$.

In order to prove that $(A, L)$ is an automatic structure with uniqueness for $G B R^{*}(T ; \beta, \gamma ; u)$ we have to prove that, for each generator $k \in A$ the language $L_{k}$ is regular. To prove that $L_{y}, L_{z}, L_{a}$ and $L_{b}$ are regular we observe that

$$
\begin{aligned}
& \left(z^{m} a^{n} \overline{t_{i}} b^{p} y^{q}\right) y=z^{m} a^{n} \overline{t_{i}} b^{p} y^{q+1}, \\
& \left(z^{m} a^{n} \overline{t_{i}} b^{p} y^{q}\right) z= \begin{cases}z^{m} a^{n} \overline{t_{i}} b^{p} y^{q-1} & \text { if } q \geq 1, \\
z^{m+1}\left(\overline{t_{i}} \gamma\right) & \text { if } q=0,\end{cases} \\
& \left(z^{m} a^{n} \overline{t_{i}} b^{p} y^{q}\right) a= \begin{cases}z^{m} a^{n}\left(\overline{t_{i}}\left(\left(u^{-1} \gamma^{q-1}\right) \beta^{p}\right)\right) b^{p} y^{q} & \text { if } q \geq 1, \\
z^{m} a^{n} \overline{i_{i}} b^{p-1} & \text { if } q=0, p \geq 1, \\
z^{m} a^{n+1}\left(\overline{t_{i}} \beta\right) & \text { if } q=p=0,\end{cases} \\
& \left(z^{m} a^{n} \overline{t_{i}} b^{p} y^{q}\right) b= \begin{cases}z^{m} a^{n}\left(\overline{t_{i}}\left(\left(u \gamma^{q-1}\right) \beta^{p}\right)\right) b^{p} y^{q} & \text { if } q \geq 1, \\
z^{m} a^{n} \overline{t_{i}} b^{p+1} & \text { if } q=0,\end{cases}
\end{aligned}
$$

and so we can write

$$
L_{y}=\bigcup_{i=1}^{l}\left\{\left(z^{m} a^{n} \overline{t_{i}} b^{p} y^{q}, z^{m} a^{n} \overline{t_{i}} b^{p} y^{q+1}\right) \delta_{A}: m, n, p, q \in \mathbb{N}^{0}\right\}
$$




$$
=\bigcup_{i=1}^{l}\left(\{(z, z)\}^{*} \cdot\{(a, a)\}^{*} \cdot\left\{\left(\overline{t_{i}}, \overline{t_{i}}\right)\right\}^{*} \cdot\{(b, b)\}^{*} \cdot\{(y, y)\}^{*} \cdot\{(\$, y)\}\right)
$$

which is a regular language. We have

$$
\begin{aligned}
L_{z}= & \bigcup_{i=1}^{l}\left\{\left(z^{m} a^{n} \overline{t_{i}} b^{p} y^{q}, z^{m} a^{n} \overline{t_{i}} b^{p} y^{q-1}\right) \delta_{A}: m, n, p \in \mathbb{N}^{0}, q \geq 1\right\} \\
& \cup \bigcup_{i=1}^{l}\left\{\left(z^{m} \overline{t_{i}}, z^{m+1}\left(\overline{t_{i}} \gamma\right)\right) \delta_{A}: m \in \mathbb{N}^{0}\right\} \\
= & \bigcup_{i=1}^{l}\left(\{(z, z)\}^{*} \cdot\{(a, a)\}^{*} \cdot\left\{\left(\overline{t_{i}}, \overline{t_{i}}\right)\right\}^{*} \cdot\{(b, b)\}^{*} \cdot\{(y, y)\}^{*} \cdot\{(y, \$)\}\right) \\
& \cup \bigcup_{i=1}^{l}\left(\{(z, z)\}^{*} \cdot\left\{\left(\overline{t_{i}}, z\right)\left(\$, \overline{t_{i} \gamma}\right)\right\}\right),
\end{aligned}
$$

and we conclude that $L_{z}$ is a regular language. Now we consider the language $L_{a}$

$$
\begin{aligned}
L_{a}= & \bigcup_{i=1}^{l}\left\{\left(z^{m} a^{n} \overline{t_{i}} b^{p} y^{q}, z^{m} a^{n}\left(\overline{t_{i}}\left(\left(u^{-1} \gamma^{q-1}\right) \beta^{p}\right)\right) b^{p} y^{q}\right) \delta_{A}: m, n, p \in \mathbb{N}^{0}, q \geq 1\right\} \\
& \cup \bigcup_{i=1}^{l}\left\{\left(z^{m} a^{n} \overline{t_{i}} b^{p}, z^{m} a^{n} \overline{t_{i}} b^{p-1}\right) \delta_{A}: m, n \in \mathbb{N}^{0}, p \geq 1\right\} \\
& \cup \bigcup_{i=1}^{l}\left\{\left(z^{m} a^{n} \overline{t_{i}}, z^{m} a^{n+1}\left(\overline{t_{i} \beta}\right)\right) \delta_{A}: m, n \in \mathbb{N}^{0}\right\} .
\end{aligned}
$$

Since $T$ is finite the set $H_{1}^{*}$ is finite as well. So $\left\{\left(u^{-1} \gamma^{q-1}\right) \beta^{p},\left(u \gamma^{q-1}\right) \beta^{p}: p, q \in\right.$ $\left.\mathbb{N}^{0}, q \geq 1\right\}$ is finite. Then we get

$$
\begin{aligned}
L_{a}= & \bigcup_{i=1}^{l}\left(\{(z, z)\}^{*} \cdot\{(a, a)\}^{*} \cdot\left\{\left(\overline{t_{i}}, \overline{t_{i}}\right)\left(\$,\left(\left(u^{-1} \gamma^{q-1}\right) \beta^{p}\right)\right)\right\} \cdot\{(b, b)\}^{*} \cdot\{(y, y)\}^{+}\right) \\
& \cup \bigcup_{i=1}^{l}\left(\{(z, z)\}^{*} \cdot\{(a, a)\}^{*} \cdot\left\{\left(\overline{t_{i}}, \overline{t_{i}}\right)\right\} \cdot\{(b, b)\}^{*} \cdot\{(b, \$)\}\right) \\
& \cup \bigcup_{i=1}^{l}\left(\{(z, z)\}^{*} \cdot\{(a, a)\}^{*} \cdot\left\{\left(\overline{t_{i}}, a\right)\left(\$, \overline{t_{i} \beta}\right)\right\}\right)
\end{aligned}
$$

which is a finite union of regular languages and so is regular.

$$
L_{b}=\bigcup_{i=1}^{l}\left\{\left(z^{m} a^{n} \overline{t_{i}} b^{p} y^{q}, z^{m} a^{n}\left(\overline{t_{i}}\left(\left(u \gamma^{q-1}\right) \beta^{p}\right)\right) b^{p} y^{q}\right) \delta_{A}: m, n, p \in \mathbb{N}^{0}, q \geq 1\right\}
$$




$$
\begin{aligned}
& \cup \bigcup_{i=1}^{l}\left\{\left(z^{m} a^{n} \overline{t_{i}} b^{p}, z^{m} a^{n} \overline{t_{i}} b^{p+1}\right) \delta_{A}: m, n, p \in \mathbb{N}^{0}\right\} \\
= & \bigcup_{i=1}^{l}\left(\{(z, z)\}^{*} \cdot\{(a, a)\}^{*} \cdot\left\{\left(\overline{t_{i}}, \overline{t_{i}}\right)\left(\$,\left(\left(u \gamma^{q-1}\right) \beta^{p}\right)\right)\right\} \cdot\{(b, b)\}^{*} \cdot\{(y, y)\}^{+}\right) \\
& \cup \bigcup_{i=1}^{l}\left(\{(z, z)\}^{*} \cdot\{(a, a)\}^{*} \cdot\left\{\left(\overline{t_{i}}, \overline{t_{i}}\right)\right\} \cdot\{(b, b)\}^{*} \cdot\{(\$, b)\}\right),
\end{aligned}
$$

and we conclude that $L_{b}$ is a regular language as well.

Now for $\bar{t} \in \bar{T}$ we have

$$
\left(z^{m} a^{n} \overline{t_{i}} b^{p} y^{q}\right) \bar{t}= \begin{cases}z^{m} a^{n} \overline{t_{i}}\left(\left(t \gamma^{q}\right) \beta^{p}\right) b^{p} y^{q} & \text { if } q \geq 1, \\ z^{m} a^{n} \overline{t_{i}}\left(t \beta^{p}\right) b^{p} & \text { if } q=0, p \geq 1 .\end{cases}
$$

Since $T$ is finite the sets $\left\{\left(t \gamma^{q}\right) \beta^{p}: p, q \in \mathbb{N}^{0}, q \geq 1\right\}$ and $\left\{t \beta^{p}: p \in \mathbb{N}^{0}\right\}$ are finite as well. Thus we have

$$
\begin{aligned}
L_{\bar{t}}= & \bigcup_{i=1}^{l}\left\{\left(z^{m} a^{n} \overline{t_{i}} b^{p} y^{q}, z^{m} a^{n} \overline{t_{i}}\left(\left(t \gamma^{q}\right) \beta^{p}\right) b^{p} y^{q}\right) \delta_{A}: m, n, p \in \mathbb{N}^{0}, q \geq 1\right\} \\
& \cup \bigcup_{i=1}^{l}\left\{\left(z^{m} a^{n} \overline{t_{i}} b^{p}, z^{m} a^{n} \overline{t_{i}}\left(t \beta^{p}\right) b^{p}\right) \delta_{A}: m, n \in \mathbb{N}^{0}, p \geq 1\right\} \\
= & \left.\bigcup_{i=1}^{l}\{(z, z)\}^{*} \cdot\{(a, a)\}^{*} \cdot\left\{\left(\overline{t_{i}}, \overline{t_{i}}\right)\left(\$,\left(\left(t \gamma^{q}\right) \beta^{p}\right)\right)\right\} \cdot\{(b, b)\}^{*} \cdot\{(y, y)\}^{+}\right) \\
& \cup \bigcup_{i=1}^{l}\left(\{(z, z)\}^{*} \cdot\{(a, a)\}^{*} \cdot\left\{\left(\overline{t_{i}}, \overline{t_{i}}\right)\left(\$, t \beta^{p}\right)\right\} \cdot\{(b, b)\}^{+}\right)
\end{aligned}
$$

which is a finite union of regular languages and so is regular.

Hence the result.

Now on we assume that $T$ is an automatic monoid and we fix an automatic structure $(X, K)$ with uniqueness for $T$, where $X=\left\{x_{1}, \cdots, x_{n}\right\}$ is a set of semigroup generators for $T$ with respect to the homomorphism

$$
\phi: X^{+} \rightarrow T \text {. }
$$

We define the alphabet

$$
A=\{y, z, a, b\} \cup X
$$

to be a set of semigroup generators for $G B R^{*}(T ; \gamma, \beta ; u)$ with respect to the homomorphism

$$
\begin{aligned}
\psi: A^{+} & \rightarrow G B R^{*}(T ; \gamma, \beta ; u) \\
x_{i} & \mapsto\left(0,0, x_{i} \phi, 0,0\right), \\
y & \mapsto\left(0,0,1_{T}, 0,1\right),
\end{aligned}
$$




$$
\begin{aligned}
z & \mapsto\left(1,0,1_{T}, 0,0\right), \\
a & \mapsto\left(0,1,1_{T}, 0,0\right), \\
b & \mapsto\left(0,0,1_{T}, 1,0\right),
\end{aligned}
$$

and the regular language

$$
L=\left\{z^{m} a^{n} w b^{p} y^{q}: w \in K ; m, n, p, q \in \mathbb{N}^{0}\right\}
$$

on $A^{+}$, which is a set of unique normal forms for $G B R^{*}(T ; \gamma, \beta ; u)$, since we have $\left(z^{m} a^{n} w b^{p} y^{q}\right) \psi=(m, n, w \phi, p, q)$ for $w \in K, m, n, p, q \in \mathbb{N}^{0}$. As usual, to simplify notation, we will avoid explicit use of the homomorphisms $\psi$ and $\phi$, associated with the generating sets, and it will be clear from the context whenever a word $w \in X^{+}$ is being identified with an element of $T$, with an element of $G B R^{*}(T ; \gamma, \beta ; u)$ or considered as a word. In particular, for a word $w \in X^{+}$we write $w \theta$ instead of $(w \phi) \theta$, seeing $\theta$ also as a homomorphism $\theta: X^{+} \rightarrow T$, and we will often write $(m, n, w, p, q)$ instead of $(m, n, w \phi, p, q)$ for $m, n, p, q \in \mathbb{N}^{0}$.

To show that $G B R^{*}(T ; \gamma, \beta ; u)$ has automatic structure $(A, L)$, the languages

$$
\begin{aligned}
L_{y}= & \left\{\left(z^{m} a^{n} w b^{p} y^{q}, z^{m} a^{n} w b^{p} y^{q+1}\right) \delta_{A}: w \in K ; m, n, p, q \in \mathbb{N}^{0}\right\}, \\
L_{z}= & \left\{\left(z^{m} a^{n} w b^{p} y^{q}, z^{m} a^{n} w b^{p} y^{q-1}\right) \delta_{A}: w \in K ; m, n, p \in \mathbb{N}^{0}, q \geq 1\right\} \\
& \cup\left\{\left(z^{m} w_{1}, z^{m+1} w_{2}\right) \delta_{A}: w_{1}, w_{2} \in K ; m \in \mathbb{N}^{0} ; w_{2}=w_{1} \gamma\right\}, \\
L_{a}= & \left\{\left(z^{m} a^{n} w_{1} b^{p} y^{q}, z^{m} a^{n} w_{2} b^{p} y^{q}\right) \delta_{A}: w_{1}, w_{2} \in K ; m, n, p \in \mathbb{N}^{0}, q \geq 1 ;\right. \\
& \left.w_{2}=w_{1}\left(\left(u^{-1} \gamma^{q-1}\right) \beta^{p}\right)\right\} \\
& \cup\left\{\left(z^{m} a^{n} w b^{p}, z^{m} a^{n} w b^{p-1}\right) \delta_{A}: w \in K ; m, n \in \mathbb{N}^{0}, p \geq 1\right\} \\
& \cup\left\{\left(z^{m} a^{n} w_{1}, z^{m} a^{n+1} w_{2}\right) \delta_{A}: w_{1}, w_{2} \in K ; m, n \in \mathbb{N}^{0} ; w_{2}=w_{1} \beta\right\}, \\
L_{b}= & \left\{\left(z^{m} a^{n} w_{1} b^{p} y^{q}, z^{m} a^{n} w_{2} b^{p} y^{q}\right) \delta_{A}: w_{1}, w_{2} \in K ; m, n, p \in \mathbb{N}^{0}, q \geq 1 ;\right. \\
& \left.w_{2}=w_{1}\left(\left(u \gamma^{q-1}\right) \beta^{p}\right)\right\} \\
& \cup\left\{\left(z^{m} a^{n} w b^{p}, z^{m} a^{n} w b^{p+1}\right) \delta_{A}: w \in K ; m, n, p \in \mathbb{N}^{0}\right\}, \\
= & \left\{\left(z^{m} a^{n} w_{1} b^{p} y^{q}, z^{m} a^{n} w_{2} b^{p} y^{q}\right) \delta_{A}:\left(w_{1}, w_{2}\right) \delta_{X} \in K_{\left(x_{r} \gamma^{q}\right) \beta^{p}} ;\right. \\
& \left.m, n, p, q \in \mathbb{N}^{0},\left(x_{r} \in X\right)\right\},
\end{aligned}
$$

must be regular. We note that the language $L_{y}$ is regular, since we have

$$
L_{y}=\{(z, z)\}^{*} \cdot\{(a, a)\}^{*} \cdot\left\{(w, w) \delta_{X}: w \in K\right\} \cdot\{(b, b)\}^{*} \cdot\{(y, y)\}^{*} \cdot\{(\$, y)\},
$$

but there is no obvious reason why the languages $L_{z}, L_{a}, L_{b}$ and $L_{x_{r}}$ should also be regular. Hence we will consider particular situations where $(A, L)$ is an automatic structure for $G B R^{*}(T ; \gamma, \beta ; u)$. We will use the notion of padded product of languages and the following result. The proof of the following result can be found in [6. Now we fix an alphabet $A$, and take two regular languages $M, N$ in $\left(A^{*} \times A^{*}\right) \delta$. Then the padded product of languages $M$ and $N$ is

$$
M \odot N=\left\{\left(w_{1} w_{1}^{\prime}, w_{2} w_{2}^{\prime}\right) \delta:\left(w_{1}, w_{2}\right) \delta \in M,\left(w_{1}^{\prime}, w_{2}^{\prime}\right) \delta \in N\right\} .
$$

The result is as follows. 
Lemma 5. Let $A$ be an alphabet and let $M, N$ be regular languages on $\left(A^{*} \times A^{*}\right) \delta$. If there exists a constant $C$ such that for any two words $w_{1}, w_{2} \in A^{*}$ we have

$$
\left(w_{1}, w_{2}\right) \delta \in M \Rightarrow|| w_{1}|-| w_{2}|| \leq C,
$$

then the language $M \odot N$ is regular.

Now we give our result.

Theorem 6. If $T$ is an automatic monoid and $\gamma, \beta: T \rightarrow H_{1}^{*} ; t \mapsto 1_{T}$ then $\operatorname{GBR}^{*}(T ; \gamma, \beta ; u)$ is automatic.

Proof. To show that the pair $(A, L)$ defined by (1) and (2) is an automatic structure for $G B R^{*}(T ; \gamma, \beta ; u)$, we have to prove that the languages $L_{z}, L_{a}, L_{b}$ and $L_{x}$ $(x \in X)$ are regular. But now we denote by $w_{1_{T}}$ the unique word in $K$ representing $1_{T}$. Then we have

$$
\begin{aligned}
L_{z}= & \left\{\left(z^{m} a^{n} w b^{p} y^{q}, z^{m} a^{n} w b^{p} y^{q-1}\right) \delta_{A}: w \in K ; m, n, p \in \mathbb{N}^{0}, q \geq 1\right\} \\
& \cup\left\{\left(z^{m} w, z^{m+1} w_{1_{T}}\right) \delta_{A}: w \in K ; m \in \mathbb{N}^{0}\right\} \\
= & \left(\{(z, z)\}^{*} \cdot\{(a, a)\}^{*} \cdot\left\{(w, w) \delta_{X}: w \in K\right\} \cdot\{(b, b)\}^{*} \cdot\{(y, y)\}^{*} \cdot\{(y, \$)\}\right) \\
& \cup\left(\{(z, z)\}^{*} \odot\left(K \times\left\{w_{1_{T}}\right\}\right) \delta_{X}\right)
\end{aligned}
$$

and

$$
\begin{aligned}
L_{a}= & \left\{\left(z^{m} a^{n} w b^{p} y^{q}, z^{m} a^{n} w b^{p} y^{q}\right) \delta_{A}: w \in K ; m, n, p \in \mathbb{N}^{0}, q \geq 1\right\} \\
& \cup\left\{\left(z^{m} a^{n} w b^{p}, z^{m} a^{n} w b^{p-1}\right) \delta_{A}: w \in K ; m, n \in \mathbb{N}^{0}, p \geq 1\right\} \\
& \cup\left\{\left(z^{m} a^{n} w, z^{m} a^{n} w_{1_{T}}\right) \delta_{A}: w \in K ; m, n \in \mathbb{N}^{0}\right\} \\
= & \left(\{(z, z)\}^{*} \cdot\{(a, a)\}^{*} \cdot\left\{(w, w) \delta_{X}: w \in K\right\} \cdot\{(b, b)\}^{*} \cdot\{(y, y)\}^{+}\right) \\
& \cup\{(z, z)\}^{*} \cdot\{(a, a)\}^{*} \cdot\left\{(w, w) \delta_{X}: w \in K\right\} \cdot\{(b, b)\}^{*} \cdot\{(b, \$)\} \\
& \cup\left(\left(\{(z, z)\}^{*} \cdot\{(a, a)\}^{*}\right) \odot\left(K \times\left\{w_{1_{T}}\right\}\right) \delta_{X}\right),
\end{aligned}
$$

which are regular languages by Lemma 5 . Now we consider the language $L_{b}$ and then we have

$$
\begin{aligned}
L_{b}= & \left\{\left(z^{m} a^{n} w b^{p} y^{q}, z^{m} a^{n} w b^{p} y^{q}\right) \delta_{A}: w \in K ; m, n, p \in \mathbb{N}^{0}, q \geq 1\right\} \\
& \cup\left\{\left(z^{m} a^{n} w b^{p}, z^{m} a^{n} w b^{p+1}\right) \delta_{A}: w \in K ; m, n, p \in \mathbb{N}^{0}\right\} \\
= & \left(\{(z, z)\}^{*} \cdot\{(a, a)\}^{*} \cdot\left\{(w, w) \delta_{X}: w \in K\right\} \cdot\{(b, b)\}^{*} \cdot\{(y, y)\}^{+}\right) \\
& \cup\left(\{(z, z)\}^{*} \cdot\{(a, a)\}^{*} \cdot\left\{(w, w) \delta_{X}: w \in K\right\} \cdot\{(b, b)\}^{*} \cdot\{(\$, b)\}\right),
\end{aligned}
$$

which is a regular language. Since, for any $z^{m} a^{n} w b^{p} y^{q} \in L$ with $q \geq 1$, we have

$$
\left(z^{m} a^{n} w b^{p} y^{q}\right) x=z^{m} a^{n} w b^{p} y^{q},
$$

and for $z^{m} a^{n} w b^{p} \in L$ with $p \geq 1$, we have

$$
\left(z^{m} a^{n} w b^{p}\right) x=z^{m} a^{n} w b^{p},
$$

and for $z^{m} a^{n} w \in L$ we have

$$
\left(z^{m} a^{n} w\right) x=z^{m} a^{n} w x
$$


we get

$$
\begin{aligned}
L_{x}= & \left\{\left(z^{m} a^{n} w b^{p} y^{q}, z^{m} a^{n} w b^{p} y^{q}\right) \delta_{A}: w \in K ; m, n, p \in \mathbb{N}^{0}, q \geq 1\right\} \\
& \cup\left\{\left(z^{m} a^{n} w b^{p}, z^{m} a^{n} w b^{p}\right) \delta_{A}: w \in K ; m, n \in \mathbb{N}^{0}, p \geq 1\right\} \\
& \cup\left\{\left(z^{m} a^{n} w_{1}, z^{m} a^{n} w_{2}\right) \delta_{A}:\left(w_{1}, w_{2}\right) \delta_{X} \in K_{x} ; m, n \in \mathbb{N}^{0}\right\} \\
= & \left(\{(z, z)\}^{*} \cdot\{(a, a)\}^{*} \cdot\left\{(w, w) \delta_{X}: w \in K\right\} \cdot\{(b, b)\}^{*} \cdot\{(y, y)\}^{+}\right) \\
& \cup\left(\{(z, z)\}^{*} \cdot\{(a, a)\}^{*} \cdot\left\{(w, w) \delta_{X}: w \in K\right\} \cdot\{(b, b)\}^{+}\right) \\
& \cup\left(\{(z, z)\}^{*} \cdot\{(a, a)\}^{*} \cdot K_{x}\right) .
\end{aligned}
$$

Hence $L_{x}$ is a regular language and so $G B R^{*}(T ; \gamma, \beta ; u)$ is automatic.

Theorem 7. If $T$ is an automatic monoid and $\gamma, \beta$ are identity homomorphisms of $T$ then $G B R^{*}(T ; \gamma, \beta ; u)$ is automatic.

Proof. To show that the pair $(A, L)$ defined by (1) and (2) is an automatic structure for $G B R^{*}(T ; \gamma, \beta ; u)$ we have to prove that the languages $L_{z}, L_{a}, L_{b}$ and $L_{x}(x \in X)$ are regular. To do that we have

$$
\begin{aligned}
L_{z}= & \left\{\left(z^{m} a^{n} w b^{p} y^{q}, z^{m} a^{n} w b^{p} y^{q-1}\right) \delta_{A}: w \in K ; m, n, p \in \mathbb{N}^{0}, q \geq 1\right\} \\
& \cup\left\{\left(z^{m} w, z^{m+1} w\right) \delta_{A}: w \in K ; m \in \mathbb{N}^{0}\right\} \\
= & \left(\{(z, z)\}^{*} \cdot\{(a, a)\}^{*} \cdot\left\{(w, w) \delta_{X}: w \in K\right\} \cdot\{(b, b)\}^{*} \cdot\{(y, y)\}^{*} \cdot\{(y, \$)\}\right) \\
& \cup\left(\left(\{(z, z)\}^{*} \cdot\{(\$, y)\}\right) \odot\left\{(w, w) \delta_{X}: w \in K\right\}\right),
\end{aligned}
$$

and

$$
\begin{aligned}
L_{a}= & \left\{\left(z^{m} a^{n} w b^{p} y^{q}, z^{m} a^{n} w u^{-1} b^{p} y^{q}\right) \delta_{A}: w, u^{-1} \in K ; m, n, p \in \mathbb{N}^{0}, q \geq 1\right\} \\
& \cup\left\{\left(z^{m} a^{n} w b^{p}, z^{m} a^{n} w b^{p-1}\right) \delta_{A}: w \in K ; m, n \in \mathbb{N}^{0}, p \geq 1\right\} \\
& \cup\left\{\left(z^{m} a^{n} w, z^{m} a^{n+1} w\right) \delta_{A}: w \in K ; m, n \in \mathbb{N}^{0}\right\} \\
= & \left(\{(z, z)\}^{*} \cdot\{(a, a)\}^{*} \cdot\left\{(w, w) \delta_{X}: w \in K\right\} \cdot\left\{\left(\$, u^{-1}\right) \delta_{X}: u^{-1} \in K\right\}\right. \\
& \left.\{(b, b)\}^{*} \cdot\{(y, y)\}^{+}\right) \\
& \cup\left(\{(z, z)\}^{*} \cdot\{(a, a)\}^{*} \cdot\left\{(w, w) \delta_{X}: w \in K\right\} \cdot\{(b, b)\}^{*} \cdot\{(b, \$)\}\right) \\
& \cup\left(\left(\{(z, z)\}^{*} \cdot\{(a, a)\}^{*} \cdot\{(\$, a)\}\right) \odot\left\{(w, w) \delta_{X}: w \in K\right\}\right),
\end{aligned}
$$

which are regular languages by Lemma 5 . We have

$$
\begin{aligned}
L_{b}= & \left\{\left(z^{m} a^{n} w b^{p} y^{q}, z^{m} a^{n} w u b^{p} y^{q}\right) \delta_{A}: w, u \in K ; m, n, p \in \mathbb{N}^{0}, q \geq 1\right\} \\
& \cup\left\{\left(z^{m} a^{n} w b^{p}, z^{m} a^{n} w b^{p+1}\right) \delta_{A}: w \in K ; m, n, p \in \mathbb{N}^{0}\right\} \\
= & \left(\{(z, z)\}^{*} \cdot\{(a, a)\}^{*} \cdot\left\{(w, w) \delta_{X}: w \in K\right\} \cdot\left\{(\$, u) \delta_{X}: u \in K\right\} .\right. \\
& \left.\{(b, b)\}^{*} \cdot\{(y, y)\}^{+}\right) \\
& \cup\left(\{(z, z)\}^{*} \cdot\{(a, a)\}^{*} \cdot\left\{(w, w) \delta_{X}: w \in K\right\} \cdot\{(b, b)\}^{*} \cdot\{(\$, b)\}\right),
\end{aligned}
$$

which is a regular language. Also we have

$$
L_{x}=\left\{\left(z^{m} a^{n} w_{1} b^{p} y^{q}, z^{m} a^{n} w_{2} b^{p} y^{q}\right) \delta_{A}:\left(w_{1}, w_{2}\right) \delta_{X} \in K_{x} ;\right.
$$




$$
\begin{aligned}
& \left.m, n, p, q \in \mathbb{N}^{0},(x \in X)\right\} \\
= & \{(z, z)\}^{*} \cdot\{(a, a)\}^{*} \cdot K_{x} \cdot\{(b, b)\}^{*} \cdot\{(y, y)\}^{*}
\end{aligned}
$$

which is a regular language. So $(A, L)$ is an automatic structure for $G B R^{*}(T ; \gamma, \beta ; u)$.

A semigroup $T$ is called of finite geometric type (fgt) (see [21]) if for every $t_{1} \in T$, there exists $k \in \mathbb{N}$ such that the equation $x t_{1}=t_{2}$ has at most $k$ solutions for every $t_{2} \in T$.

To prove the next theorem we need the following two lemmas which were proved in 6.

Lemma 8. Let $T$ be a finite geometric type monoid with an automatic structure with uniqueness $(X, K)$. Then for every $w \in X^{+}$there is a constant $C$ such that $\left(w_{1}, w_{2}\right) \delta_{X} \in K_{w}$ implies ||$w_{1}|-| w_{2}||<C$.

Lemma 9. Let $S$ be a finite semigroup, $X$ be a finite set and $\psi: X^{+} \rightarrow S$ be a surjective homomorphism. For any $s \in S$ the set $s \psi^{-1}$ is a regular language.

Theorem 10. Let $T$ be a finite geometric type automatic monoid and let $\gamma, \beta: T \rightarrow$ $H_{1}^{*}$ be homomorphisms. If $T \gamma, T \beta$ are finite then $G B R^{*}(T ; \gamma, \beta ; u)$ is automatic.

Proof. We will prove that the pair $(A, L)$ defined by (1) and (2) is an automatic structure for $G B R^{*}(T ; \gamma, \beta ; u)$. To do that we have

$$
\begin{aligned}
L_{z}= & \left\{\left(z^{m} a^{n} w b^{p} y^{q}, z^{m} a^{n} w b^{p} y^{q-1}\right) \delta_{A}: w \in K ; m, n, p \in \mathbb{N}^{0}, q \geq 1\right\} \\
& \cup\left\{\left(z^{m} w_{1}, z^{m+1} w_{2}\right) \delta_{A}: w_{1}, w_{2} \in K ; m \in \mathbb{N}^{0} ; w_{2}=w_{1} \gamma\right\} .
\end{aligned}
$$

It is seen that the language

$$
\begin{array}{r}
\left\{\left(z^{m} a^{n} w b^{p} y^{q}, z^{m} a^{n} w b^{p} y^{q-1}\right) \delta_{A}: w \in K ; m, n, p \in \mathbb{N}^{0}, q \geq 1\right\}= \\
\{(z, z)\}^{*} \cdot\{(a, a)\}^{*} \cdot\left\{(w, w) \delta_{X}: w \in K\right\} \cdot\{(b, b)\}^{*} \cdot\{(y, y)\}^{*} \cdot\{(y, \$)\}
\end{array}
$$

is regular. Thus we just have to prove that the language

$$
M=\left\{\left(z^{m} w_{1}, z^{m+1} w_{2}\right) \delta_{A}: w_{1}, w_{2} \in K ; m \in \mathbb{N}^{0} ; w_{2}=w_{1} \gamma\right\}
$$

is also regular. For any $t \in T \gamma$, let $w_{t}$ be the unique word in $K$ representing $t$. Let

$$
\begin{aligned}
N & =\left\{\left(w_{1}, w_{2}\right) \delta_{X}: w_{1}, w_{2} \in K ; w_{2}=w_{1} \gamma\right\} \\
& =\bigcup_{t \in T \gamma}\left\{\left(w_{1}, w_{2}\right) \delta_{X}: w_{1}, w_{2} \in K ; w_{2}=w_{1} \gamma=t\right\} \\
& =\bigcup_{t \in T \gamma}\left\{\left(w_{1}, w_{t}\right) \delta_{X}: w_{1} \in K ; w_{1} \in\left(t \gamma^{-1}\right) \phi^{-1}\right\} \\
& =\bigcup_{t \in T \gamma}\left(\left(\left(t \gamma^{-1}\right) \phi^{-1} \cap K\right) \times\left\{w_{t}\right\}\right) \delta_{X} .
\end{aligned}
$$


We can define $\psi: X^{+} \rightarrow T \gamma ; w \mapsto w \phi \gamma$ and, since $T \gamma$ is finite, for any $t \in T \gamma$, we can apply Lemma 9 and conclude that $\left(t \gamma^{-1}\right) \phi^{-1}=t \psi^{-1}$ is regular. Therefore, $N$ is a regular language. By Lemma 5 the language

$$
\begin{aligned}
M & =\left\{\left(z^{m} w_{1}, z^{m+1} w_{2}\right) \delta_{A}:\left(w_{1}, w_{2}\right) \delta_{X} \in N ; m \in \mathbb{N}^{0}\right\} \\
& =\left(\{(z, z)\}^{*} \cdot\{(\$, z)\}\right) \odot N
\end{aligned}
$$

is regular. Now we will show that the language

$$
\begin{aligned}
L_{a}= & \left\{\left(z^{m} a^{n} w_{1} b^{p} y^{q}, z^{m} a^{n} w_{2} b^{p} y^{q}\right) \delta_{A}: w_{1}, w_{2} \in K ; m, n, p \in \mathbb{N}^{0}, q \geq 1 ;\right. \\
& \left.w_{2}=w_{1}\left(\left(u^{-1} \gamma^{q-1}\right) \beta^{p}\right)\right\} \\
& \cup\left\{\left(z^{m} a^{n} w b^{p}, z^{m} a^{n} w b^{p-1}\right) \delta_{A}: w \in K ; m, n \in \mathbb{N}^{0}, p \geq 1\right\} \\
& \cup\left\{\left(z^{m} a^{n} w_{1}, z^{m} a^{n+1} w_{2}\right) \delta_{A}: w_{1}, w_{2} \in K ; m, n \in \mathbb{N}^{0} ; w_{2}=w_{1} \beta\right\}
\end{aligned}
$$

is regular. Since the language

$$
\begin{array}{r}
\left\{\left(z^{m} a^{n} w b^{p}, z^{m} a^{n} w b^{p-1}\right) \delta_{A}: w \in K ; m, n \in \mathbb{N}^{0}, p \geq 1\right\}= \\
\{(z, z)\}^{*} \cdot\{(a, a)\}^{*} \cdot\left\{(w, w) \delta_{X}: w \in K\right\} \cdot\{(b, b)\}^{*} \cdot\{(b, \$)\}
\end{array}
$$

is regular, we have to prove that

$$
\begin{aligned}
M_{1}= & \left\{\left(z^{m} a^{n} w_{1} b^{p} y^{q}, z^{m} a^{n} w_{2} b^{p} y^{q}\right) \delta_{A}: w_{1}, w_{2} \in K ; m, n, p \in \mathbb{N}^{0}, q \geq 1 ;\right. \\
& \left.w_{2}=w_{1}\left(\left(u^{-1} \gamma^{q-1}\right) \beta^{p}\right)\right\},
\end{aligned}
$$

and

$$
M_{2}=\left\{\left(z^{m} a^{n} w_{1}, z^{m} a^{n+1} w_{2}\right) \delta_{A}: w_{1}, w_{2} \in K ; m, n \in \mathbb{N}^{0} ; w_{2}=w_{1} \beta\right\}
$$

are regular. It is seen that the language

$$
\begin{aligned}
M_{1}= & \left\{\left(z^{m} a^{n} w_{1} b^{p} y^{q}, z^{m} a^{n} w_{2} b^{p} y^{q}\right) \delta_{A}: w_{1}, w_{2} \in K ; m, n, p \in \mathbb{N}^{0}, q \geq 1\right. \\
& \left.w_{2}=w_{1}\left(\left(u^{-1} \gamma^{q-1}\right) \beta^{p}\right)\right\} \\
= & \{(z, z)\}^{*} \cdot\{(a, a)\}^{*} \cdot\left\{\left(w_{1}, w_{1}\right) \delta_{X}: w_{1} \in K\right\} \cdot\left\{\left(\$,\left(u^{-1} \gamma^{q-1}\right) \beta^{p}\right)\right\} \\
& \{(b, b)\}^{*} \cdot\{(y, y)\}^{*}
\end{aligned}
$$

is regular. Now for any $t \in T \beta$, let $w_{t}$ be the unique word in $K$ representing $t$. Let

$$
\begin{aligned}
N_{2} & =\left\{\left(w_{1}, w_{2}\right) \delta_{X}: w_{1}, w_{2} \in K ; w_{2}=w_{1} \beta\right\} \\
& =\bigcup_{t \in T \beta}\left\{\left(w_{1}, w_{2}\right) \delta_{X}: w_{1}, w_{2} \in K ; w_{2}=w_{1} \beta=t\right\} \\
& =\bigcup_{t \in T \beta}\left\{\left(w_{1}, w_{t}\right) \delta_{X}: w_{1} \in K ; w_{1} \in\left(t \beta^{-1}\right) \phi^{-1}\right\} \\
& =\bigcup_{t \in T \beta}\left(\left(\left(t \beta^{-1}\right) \phi^{-1} \cap K\right) \times\left\{w_{t}\right\}\right) \delta_{X} .
\end{aligned}
$$

We can define $\psi_{2}: X^{+} \rightarrow T \beta ; w \mapsto w \phi \beta$ and, since $T \beta$ is finite, for any $t \in T \beta$, we can apply Lemma 9 and conclude that $\left(t \beta^{-1}\right) \phi^{-1}=t \psi^{-1}$ is regular. Therefore, 
$N_{2}$ is a regular language. By Lemma 5, we have that the language

$$
\begin{aligned}
M_{2} & =\left\{\left(z^{m} a^{n} w_{1}, z^{m} a^{n+1} w_{2}\right) \delta_{A}:\left(w_{1}, w_{2}\right) \delta_{X} \in N_{2} ; m, n \in \mathbb{N}^{0}\right\} \\
& =\left(\{(z, z)\}^{*} \cdot\{(a, a)\}^{*} \cdot\{(\$, a)\}\right) \odot N_{2}
\end{aligned}
$$

is regular.

Now we will prove that the language

$$
\begin{aligned}
L_{b}= & \left\{\left(z^{m} a^{n} w_{1} b^{p} y^{q}, z^{m} a^{n} w_{2} b^{p} y^{q}\right) \delta_{A}: w_{1}, w_{2} \in K ; m, n, p \in \mathbb{N}^{0}, q \geq 1 ;\right. \\
& \left.w_{2}=w_{1}\left(\left(u \gamma^{q-1}\right) \beta^{p}\right)\right\} \\
& \cup\left\{\left(z^{m} a^{n} w b^{p}, z^{m} a^{n} w b^{p+1}\right) \delta_{A}: w \in K ; m, n, p \in \mathbb{N}^{0}\right\}
\end{aligned}
$$

is regular. Since the languages

$$
\begin{array}{r}
\left\{\left(z^{m} a^{n} w b^{p}, z^{m} a^{n} w b^{p+1}\right) \delta_{A}: w \in K ; m, n, p \in \mathbb{N}^{0}\right\}= \\
\{(z, z)\}^{*} \cdot\{(a, a)\}^{*} \cdot\left\{(w, w) \delta_{X}: w \in K\right\} \cdot\{(b, b)\}^{*} \cdot\{(\$, b)\}
\end{array}
$$

and

$$
\begin{aligned}
& \left\{\left(z^{m} a^{n} w_{1} b^{p} y^{q}, z^{m} a^{n} w_{2} b^{p} y^{q}\right) \delta_{A}: w_{1}, w_{2} \in K, m, n, p \in \mathbb{N}^{0}, q \geq 1 ;\right. \\
& \left.w_{2}=w_{1}\left(\left(u \gamma^{q-1}\right) \beta^{p}\right)\right\}=\{(z, z)\}^{*} \cdot\{(a, a)\}^{*} \cdot\left\{\left(w_{1}, w_{1}\right) \delta_{X}: w_{1} \in K\right\} \cdot \\
& \left\{\left(\$,\left(u \gamma^{q-1}\right) \beta^{p}\right)\right\} \cdot\{(b, b)\}^{*} \cdot\{(y, y)\}^{*}
\end{aligned}
$$

are regular, $L_{b}$ is regular as well.

Now it remains to prove that the language

$$
\begin{aligned}
L_{x}= & \left\{\left(z^{m} a^{n} w_{1} b^{p} y^{q}, z^{m} a^{n} w_{2} b^{p} y^{q}\right) \delta_{A}:\left(w_{1}, w_{2}\right) \delta_{X} \in K_{\left(x \gamma^{q}\right) \beta^{p}} ;\right. \\
& \left.m, n, p, q \in \mathbb{N}^{0}(x \in X)\right\}
\end{aligned}
$$

is regular. We have

$$
L_{x}=\{(z, z)\}^{*} \cdot\{(a, a)\}^{*} \cdot\left(K_{\left(x \gamma^{q}\right) \beta^{p}} \odot\{(b, b)\}^{*}\right) \cdot\{(y, y)\}^{*} .
$$

Since $T$ is finite geometric type, by Lemma 8 there is a constant $C$ such that $\left(w_{1}, w_{2}\right) \delta_{X} \in K_{\left(x \gamma^{q}\right) \beta^{p}}$ implies $\| w_{1}|-| w_{2}||<C$, for any $p, q \in \mathbb{N}^{0}$, and thus we can apply Lemma 5 and we conclude that $L_{x}$ is a regular language.

As known, for a given construction, natural questions are:

(1) Is the class of automatic semigroups closed under this construction?

(2) If a semigroup resulting from such a construction is automatic, is the original semigroup (or are the original semigroups) automatic?

In this paper, we answered the first question "yes" under some certain situations for generalized Bruck-Reilly *-extension. But the second question is still open.

Acknowledgement. I would like to thank Prof. Dr. Ahmet Sinan Çevik for advising me to study on this subject and referees for their kind comments on the paper. 


\section{REFERENCES}

[1] Andrade, L. and Descalço, L., Martins, M. A., Automatic structures for semigroup constructions, Semigroup Forum, 76(2) (2008) 239-255.

[2] Asibong-Ibe, U., *-Bisimple type A w-semigroups-I, Semigroup Forum, 31 (1985) 99-117.

[3] Cain, A. J., Automatic semigroups and Bruck-Reilly extensions, Acta Math. Hungar., 126(12) (2010) 1-15.

[4] Campbell, C. M., Robertson, E. F., Ruškuc, N. and Thomas, R. M., Direct products of automatic semigroups, J. Austral. Math. Soc. Ser. A, 69 (2000) 19-24.

[5] Campbell, C. M., Robertson, E. F., Ruškuc, N. and Thomas, R. M., Automatic semigroups, Theoretical Computer Science, 250 (2001) 365-391.

[6] Descalço, L., Automatic semigroups: Constructions and subsemigroups, Ph.D. Thesis, University of St Andrews, 2002.

[7] Descalço, L. and Ruškuc, N., On automatic Rees matrix semigroups, Comm. Algebra, 30 (2002) 1207-1226.

[8] Epstein, D. B. A., Cannon, J. W., Holt, D. F., Levy, S. V. F., Paterson, M. S. and Thurston, W. P., Word Processing in Groups, Jones \& Bartlett (Boston, Mass.), 1992.

[9] Hoffmann, M., Automatic Semigroups, Ph.D. Thesis, University of Leicester, 2001.

[10] Hoffmann, M. and Thomas, R. M., Automaticity and commutative semigroups, Glasgow J. Math., 44 (2002) 167-176.

[11] Howie, J. M., Fundamentals of Semigroup Theory, London Mathematical Society Monographs New Series, Clarendon Press, Oxford, 1995.

[12] Hudson, J. F. P., Regular rewrite systems and automatic structures, in J. Almeida, G. M. S. Gomes and P. V. Silva, edts, Semigroups, Automata and Languages, World Scientific, Singapore, 1996, pp. 145-152.

[13] Karpuz, E. G., Çevik, A. S., Koppitz, J. and Cangül, I. N., Some fixed-point results on (generalized) Bruck-Reilly *-extensions of monoids, Fixed Point Theory and Applications, (2013) 2013: 78, doi: 10.1186/1687-1812-2013-78.

[14] Kocapinar, C., Karpuz, E., G., Ateş, F. and Çevik, A. S., Gröbner-Shirshov bases of the generalized Bruck-Reilly *-extension, Algebra Colloquium, 19 (Spec1) (2012) 813-820.

[15] Oguz, S. and Karpuz, E. G., Some semigroup classes and congruences on Bruck-Reilly and generalized Bruck-Reilly *-extensions of monoids, Asian-European Journal of Mathematics, 8(4) (2015) DOI: 10.1142/S1793557115500758.

[16] Oguz, S. and Karpuz, E. G., Finite presentability of generalized Bruck-Reilly *-extension of groups, Asian-European Journal of Mathematics, 9(4) (2016).

[17] Otto, F., On s-regular prefix-rewriting systems and automatic structures, Computing and Combinatories (Tokyo, 1999), Lecture Notes in Comput. Sci., 1627, Springer, Berlin, 1999, pp. $422-431$.

[18] Otto, F., On Dehn functions of finitely presented bi-automatic monoids, J. Austom. Lang. Comb., 5 (2000) 405-419.

[19] Otto, F., Sattler-Klein, A. and Madlener, K., Automatic monoids versus monoids with finite convergent presentations, Rewriting Techniques and Applications (Tsukuba, 1998), Lecture Notes in Comput. Sci., 1379, Springer, Berlin, 1998, pp. 32-46.

[20] Shang, Y. and Wang, L. M., *-Bisimple type A $w^{2}$-semigroups as generalized Bruck-Reilly *-extensions, Southeast Asian Bulletin of Math., 32 (2008) 343-361.

[21] Silva, P. V. and Steinberg, B., A geometric characterization of automatic monoids, Quart. J. Math., 55 (2004) 333-356. 
Current address: Eylem Güzel Karpuz: Karamanoglu Mehmetbey University, Department of Mathematics, Kamil Özdag Science Faculty, Yunus Emre Campus, 70100, Karaman-Turkey.

E-mail address: eylem.guzel@kmu.edu.tr

ORCID Address: http://orcid.org/0000-0002-7111-3462 